\title{
Gut Microbiota and Type 2 Diabetes Mellitus : What is The Link?
}

\author{
Nesma A Ibrahim
}

Internal Medicine Department, Faculty of Medicine, Ain Shams University, Cairo, Egypt

Corresponding Author Ibrahim NA

Mobile: +01007587891

Email:

dr_nesmali@yahoo.com

Key words:

Type 2 Diabetes Mellitus, Gut Microbiota

\section{Abstract:}

The rapid increase of cases of type 2 diabetes mellitus (T2DM) in the past decades has made it a widespread metabolic disorder. In addition to well-established risk factors for T2DM, including genetic predisposition, poor physical activity, fetal programming and obesity, an altered configuration of the microbial community in our gut - the microbiota - has emerged as a new candidate that may be linked to T2DM. The aim of this review is to focus the light on the role of gut microbiota as a novel key organ involved in metabolism, and discussing the putative mechanisms linking gut microbiota and T2DM, as well as the therapeutic perspective of intestinal microbiota modulation for T2DM.

\section{INTRODUCTION}

Microorganisms which colonize all surfaces of the human body that are exposed to the environment (that live on and inside humans) are collectively called the human microbiota [1]. The human microbiota consists of as many as 10 to 100 trillion microorganisms, representing at least 10 -fold more cells than exist in the human body. The main mass of microorganisms associated with humans resides in the gastrointestinal tract. The weight of the bacteria living in a human intestine is about $1.5 \mathrm{~kg}$ and make up about $50 \%$ of the fecal matter [2]. Apart from the intestinal microbiota, also the skin, oral, nasal and vaginal microbiota had been studied extensively. The human microbiota is not restricted to these sites but do also reside in for example the lungs, the blood and atherosclerotic plaques [3].

\section{MICROBIOTA COMPOSITION}

In adults, Bacteroidetes (eg, Bacteroides, Prevotella) and Firmicutes (eg, Clostridium, Enterococcus, Lactobacillus, Ruminococcus) usually dominate the intestinal microbiota, whereas Actinobacteria (eg, Bifidobacterium), Proteobacteria (eg, Helicobacter, Escherichia) and Verrucomicrobia are in considerably minor proportion.
Methanogenic archaea (represented by Methanobrevibacter smithii), eukaryotes (mainly yeast) and viruses (mainly bacteriophages) are also components of this microbiota [4].

\section{MICROBIOTA ESTABLISHMENT}

The microbial colonization of the gut begins in infants immediately after birth. Facultative anaerobes, such as enterobacteria, enterococci and lactobacilli are the first colonizers. Anaerobic microorganisms, including Bifidobacterium, Bacteroides and Clostridium establish gradually, and contribute to a progressive decrease of the facultative anaerobes to strict anaerobes ratio in time [5]. At about 3 years of age, the gut microbiota reaches a composition and diversity similar to adults and remains more or less stable over time in adulthood. New changes appear in the senescence, the microbiota of elderly people differing from the core microbiota and diversity levels of younger adults [6].

\section{FACTORS AFFECTING MICROBIOTA}

Changes are produced in our microbiota from birth to adulthood. The composition and function of the 
gut microbiota are influenced by several factors. The mode of delivery (vaginal versus Caesarian section) as well as the method of feeding (breast versus formula feeding) are influencing the gut microbiota. Both host genotype and lifestyle factors such as diet, physical activity, antibiotics, age and probably several additional but yet unidentified factors may simultaneously influence the gut microbiota [7].

\section{GUT MICROBIOTA AND METABOLISM}

The gut microbiota has gone from being considered an accompanying commensal to a «metabolic organ» [8], with functions in nutrition, immunity regulation, and systemic inflammation. The microbiota regulates, via different mechanisms, some important physiological functions of the host, such as those related to energy expenditure, satiety and glucose homeostasis. It can also act as a barrier against the establishment of food borne pathogens [9]. Growing evidence in clinical studies suggested that obese people with insulin resistance were characterized by an altered composition of gut microbiota, particularly an elevated Firmicutes/Bacteroidetes ratio compared with healthy people [10]. Furthermore, transplantation of the obese gut microbiota in animals greatly affected the energy harvest of hosts [11].

\section{THE ROLE OF GUT MICROBIOTA IN THE PATHOGENESIS OF T2DM}

\section{(I) Storage hypothesis}

Gut microbiota is involved in several intestinal biological functions such as defense against pathogens, immunity, the development of the intestinal microvilli and the degradation of non digestible polysaccharides (fermentation of resistant starch, oligosaccharides, inulin). Hence, the gut microbiota harvests energy for the host from dietary compounds ingested but not digested by the host [12]. It was found that gut microbiota conventionalisation results in a doubling of the density of capillaries in the small intestinal villus epithelium, thereby helping to promote intestinal monosaccharide absorption [13].

Backhed, et al. found that the mice raised in the absence of microorganisms (germ free) had about $40 \%$ less total body fat than mice with a normal gut microbiota. The mechanisms of the apparent weight gain implied an increase in the intestinal glucose absorption, energy extraction from non-digestible food component and concomitant higher glycemia and insulinemia, two key metabolic factors regulating lipogenesis [14]. Moreover, glucose and insulin are also known to promote hepatic de novo lipogenesis through the expression of several key enzymes such as aceyl-CoA carboxylase (ACC) and fatty acid synthase (FAS) [15].

Interestingly, a role for adipocyte lipoprotein lipase (LPL) activity was proposed. Consistent with this hypothesis, it was suggested that gut microbiota promote fat storage through a mechanism linking circulating triglycerides with suppression of the intestinal expression of an LPL inhibitor (FIAF, fasting-induced adipose factor). FIAF inhibits LPL activity, thereby reducing fatty acid release from circulating triacylglycerols. Hence, upon gut colonisation with microbiota, FIAF expression is reduced, leading to higher LPL activity and greater fat storage [14].

Also, microbiota impacts muscle metabolism and consequently influences the regulation of insulin resistance. AMP-activated kinase is an enzyme expressed in the muscle that activates glucose utilization during hypoxia and exercise [16]. It was found that germ-free mice are characterized by a very high concentration of AMP-activated kinase and its downstream targets that are involved in fatty acid oxidation in skeletal muscle favoring muscle glucose utilization and allowing the mice to resist high-fat, sugar-rich diet induced diabetes. More precisely, the gut microbiota was found to suppress AMPK-driven fatty acid oxidation in the liver and in skeletal muscle [17].

Moreover, a pathway involving short chain fatty acids (SCFAs) has been proposed. SCFAs act as signaling molecules and are specific ligands for at least two G protein-coupled receptors, GPR41 and GPR43 [18]. Samuel et al. have demonstrated that G-protein coupled receptor 41 (GPR41) knockout mice colonized with a specific fermentative microbial community resist fat mass gain compared to their wild-type littermates [19]. Therefore, this supports the idea that specific metabolites coming from the gut (i.e., SCFAs) act in a variety of ways (e.g., as energy substrates and as metabolic regulators) [20].

\section{(II) The inflammatory hypothesis}

Metabolic diseases are linked to disruption of both the innate and the adaptive immune systems. One of the mechanisms proposed to explain the crosstalk between gut microbiota, regulation of fat storage and development of obesity-related diseases is metabolic endotoxemia (i.e., increased plasma lipopolysaccharides levels) [21]. Bacterial 
lipopolysaccharides (LPS) are a component of the cell wall of gram-negative bacteria capable of triggering an inflammatory state, augment adipose inflammation and reduce insulin sensitivity, which are present in metabolic disorders [22].

It was suggested that diet may play an important role in gut permeability since LPS absorption from the gut was found to be associated with the ingestion of dietary fat [23]. A study had raised the possibility that LPS could be associated with chylomicrons within the enterocyte, being LPS then secreted from cell-associated pools in a chylomicron-dependent manner [24]. Another possibility is that dietary fat leads to paracellular leakage of LPS across the intestinal epithelium. This is supported by the observation that intestinal tight-junction integrity is impaired in obese mice and by studies in which intestinal luminal exposure to oleic acid can cause intestinal epithelial damage [25].

Gut microbiota may have a critical function in the regulation of gut permeability, contributing to endotoxemia, through the endocannabinoid system (eCB) and LPS regulatory loop [26]. On the other hand, LPS regulates the synthesis of $\mathrm{eCB}$ in macrophages [27]. Moreover, intestinal alkaline phosphatase (IAP) is known to be involved in the breakdown of dietary lipids, it also plays an important role in LPS detoxification by dephosphorylating the lipid portion of the LPS, thus acting as a host defense factor against LPS. IAP expression is not only modulated by dietary components, including fat, but also controlled by gut microbiota [28]. Interestingly, Everard and colleagues had defined the protective role of the bacterium Akkermansia (A.) muciniphila against the development of metabolic diseases [29]. The normalization of $A$. muciniphila abundance through prebiotic administration is correlated with an improved metabolic profile, reduced fatmass, metabolic endotoxemia, adipose tissue inflammation and insulin resistance [30].

These results indicate the involvement of the gut microbiota in the inception of gut barrier alterations and thus increased intestinal permeability and increased absorption of LPS, a state of metabolic endotoxemia is initiated, characterized by elevated serum LPS concentration, leading to increased activation of inflammatory pathways and impairment of the insulin signaling [31].

\section{Metabolic Endotoxemia and Insulin Resistance}

Toll-like receptors (TLRs) play an important role in the activation of innate immune responses in mammals by recognizing conserved pathogenassociated molecular patterns [32]. TLR4 is a subclass of TLRs that can be activated by LPS and by nonbacterial agonists, such as saturated fatty acids [18]. The activation of TLR4 signaling induces up-regulation of inflammatory pathways related to the induction of insulin resistance [33]. Activation of TLR4 by LPS in pre-adipocytes increases the expression of several cytokines, mainly TNF- $\alpha$ and IL-6, impairing the insulin signaling in adipocytes. Moreover, LPS can promote the expression of inducible nitric oxide synthase (iNOS), which is also known as capable of interfering with the insulin signaling [34]. Likewise, TLR2 has been shown as an important modulator of insulin resistance. It was reported that short-term inhibition of TLR2 expression using TLR2 oligonucletide antisense in dietinduced obese mice leads to increased insulin sensitivity and signaling [35]. Thus it was suggested that a signalling cascade initiated by an LPS/ TLR-4/CD14-dependent mechanism in turn activates TLR-2 expression to support innate immune system inflammatory responses [36].

Like TLRs, nucleotide oligomerization domain (NOD)-1 and -2 proteins are intracellular pattern recognition receptors that sense bacterial cell wall peptidoglycan (PGN) moieties, which induce stress and inflammation pathways [37]. Several studies have associated NOD1- and NOD2activating bacterial motifs with insulin resistance [38]. On the other hand, SCFAs were shown to affect pancreatic beta-cell function (promoting $\beta$ cell development, proliferation, and differentiation) or by indirectly increasing glucagon-like peptide1 (GLP-1) secretion from enteroendocrine L-cells. Furthermore, SCFAs reduce the release of proinflammatory cytokines by adipose tissue and weaken leukocyte activation. These antiinflammatory effects improve insulin resistance, tissue glucose uptake, and blood glucose levels [39].

\section{THERAPEUTIC PERSPECTIVE OF GUT MICROBIOTA MODULATION FOR TYPE 2 DIABETES MELLITUS}

Understanding the metabolic impact of the complex interaction between gut microbiota and the host has driven interest in manipulating microbiota in order to develop new therapeutic targets for the metabolic diseases [40].

\section{Diet and Physical activity}

Weight loss promoted by calories restricted diet and increased physical activity is associated with 
significant changes in the composition of gut microflora [30]. Recent studies in humans and rodents have demonstrated a remarkable ability of the metabolic capacity of the gut microbiota to be altered by long-term dietary patterns, indicating that the potential benefits of dietary intervention on host metabolism might very well be significant [41]. Moreover, evidence for the existence of a modulating effect of physical activity on the gut microbiota is accumulating, although mainly from rodent models. Physical activity has been shown to affect gut microbiota composition and diversity in healthy rats [42], with some results indicating that the demonstrated effects are independent of diet [43].

\section{Antibiotics}

Antibiotic treatment is another method of gut microbiota modulation. Mouse studies have shown improved glucose tolerance that was independent of weight changes, and also lower levels of circulating LPS and a lower bacterial count following antibiotic treatment, with an improved metabolic state [44]. However, the administration of antimicrobial agents, including broad spectrum antibiotics, has been proposed as a possible contributor to the obesity epidemic and the shrinking gut microbiome richness in the western world [45], it was shown that even prenatal exposure to antibiotics increases the risk of childhood obesity, perhaps through an effect on the maternal gut microbiota [46]. On the other hand, treatment with vancomycin has been shown to reduce peripheral insulin sensitivity in individuals with metabolic syndrome, whereas treatment with amoxicillin does not, reflecting the preferential targeting of Gram positive butyrate-producing bacteria by the former [47]. Although some studies indicate that antibiotics have not only short- but also long-term effects [48] on the diversity and/or configuration of the gut microbiota, further studies should be performed to investigate the effects of different antibiotics and administration routes on metabolism and T2DM [49].

\section{Bariatric surgery}

It was suggest that the more long-term health benefits of bariatric surgery may in part be due to alterations in the gut microbiota [50]. Some studies conducted on subjects submitted to surgical Roux-en-Y gastric by-pass (RYGB) reported a profound change of gut microbiota composition, related to the surgically reverted anatomy of alimentary tube [30]. The direct transit of carbohydrates to the small intestine, without the prior exposure to gastric acids, promotes the growth of Proteobacteria and Enterobacteria fermenting complex carbohydrates [51]. The increased production of metabolites deriving from oligosaccharides fermentation is well known to contribute to increased GLP-1 and peptide YY production, which contribute to reduce appetite and to improve beta-pancreatic cell function and insulin secretion [12].

\section{Manipulating the Gut Microbiota by Probiotics} Probiotics are defined as "live microorganisms that, when administered in adequate amounts, confer a health benefits on the host' [52]. Probiotics are live microorganisms administered in attempt to reconstitute the gut microbiota, namely Bifidobacterium, Lactobacillus, Saccharomyces, Streptococcus, and Enterococcus [53].

Several studies have demonstrated that probiotic strains, in particular those of the Lactobacillus and Bifidobacterium genera, exert multiple beneficial effects in subjects affected by metabolic syndrome. Indeed, they seem to promote weight loss and the reduction of visceral adiposity, to improve glucose tolerance, and to modulate intestinal low grade inflammation [30]. Molecular mechanisms involving the anti-diabetic effects of probiotics are not fully elucidated, but may be related to reduction of oxidative stress, immunomodulation, attenuation of inflammation and modification of the intestinal microbiota [54].

\section{Manipulating the Gut Microbiota by Prebiotics}

Prebiotics are defined as 'the selective stimulation of growth and/or activity(ies) of one or a limited number of microbial genus(era)/species in the gut microbiota that confer(s) health benefits to the host' [55]. The prebiotic approach dictates that non-viable food components are specifically fermented in the colon by indigenous bacteria thought to be of positive value, e.g., bifidobacteria, lactobacilli. Any food ingredient that enters the large intestine is a candidate prebiotic [56]. Prebiotics contribute to modify gut microbial composition, enhancing the growth of Bifidobacteria [57], Bacteroides, Prevotella and Roseburia [58] and promoting the relative decrease of Firmicutes [57].

Substrates that are widely accepted prebiotics include the fructans inulin and fructooligosaccharides (FOS), galacto-oligosaccharides (GOS), and lactulose [59]. Some non-digestible carbohydrates (NDCs) are recognised as prebiotics 
after they were preferentially fermented by specific types of bacteria generally considered beneficial for the host [55].

The mechanisms of action remain unclear but could be related to the regulation of intestinal mucosal biology where the intestinal mucosa was characterized by higher villi, deeper crypts, increased number of goblet cells and a thicker mucus layer on the colonic epithelium [60]. The reduction of intestinal low grade inflammation promoted by the improvement of gut barrier integrity [58] and the decrease of pro-inflammatory cytokines release, lead to an improvement of glucose tolerance and insulin sensitivity [61].

\section{Manipulating the Gut Microbiota by Fecal Transplants}

Fecal microbiota transplantation (FMT) has been utilized for over 50 years, the main principle of fecal transplant is the possibility of this procedure replacing pathogenic microbes by beneficial communities, thus restoring the gut microbiota balance and enabling the cure of the disease [40].

Vrieze et al. evaluated the effects of FMT on insulin sensitivity in individuals with metabolic syndrome. Subjects with metabolic syndrome were randomly assigned to groups set to receive small intestinal infusions from lean donors or autologous microbiota. Subjects who received infusions from lean donors were noted to have an increase in insulin sensitivity and an increase in butyrate producing intestinal microbiota 6 weeks post transfusion. This led authors to conclude that intestinal microbiota might be developed as a therapeutic agent to increase insulin sensitivity in patients with metabolic syndrome and insulin resistance [62].

\section{CONCLUSION}

Gut microbiota may play an important role in the pathogenesis of T2DM by influencing body weight, proinflammatory activity and insulin resistance. Modulating the gut microbiota through the use of probiotics, prebiotics, antibiotics, and fecal microbiota transplantation may have benefits in improving glucose metabolism and insulin resistance in the host. Although groundbreaking research in recent years has enormously expanded our understanding of the microbiotahost interplay, the numerous cellular and molecular mechanisms involved in the pathophysiological interactions await further discovery and characterization. Future studies are required to increase our understanding of the complex interplay between intestinal microbiota and the host to enable the development of new effective treatments for T2DM.

\section{REFERENCES}

1. Karlsson F, Tremaroli V, Nielsen J , Bäckhed F. Assessing the Human Gut Microbiota in Metabolic Diseases. Diabetes 2013; 62:3341-3349.

2. Zhao L. The gut microbiota and obesity: from correlation to causality. Nat Rev Microbiol 2013;11, 639-647.

3. Amar J, Lange C, Payros G, Garret C, Chabo C, Lantieri $\mathrm{O}$, et al. Blood microbiota dysbiosis is associated with the onset of cardiovascular events in a large general population: the D.E.S.I.R. study. PLoS One 2013; 8, e54461.

4. Kim BS, Jeon YS, Chun J. Current status and future promise of the human microbiome. Pediatr Gastroenterol Hepatol Nutr 2013; 16: 71-79.

5. Arboleya S, Solís G, Fernández N, de los ReyesGavilán CG and Gueimonde M. Facultative to strict anaerobes ratio in the preterm infant microbiota: a target for intervention? Gut Microbes 2012; 3: 583-588.

6. Salazar N, López P, Valdés L, Margolles A, Suárez A, Patterson AM, et al. Microbial targets for the development of functional foods accordingly with nutritional and immune parameters altered in the elderly. J Am Coll Nutr 2013; 32: 399-406.

7. Allin KH, Nielsen $\mathrm{T}$, Pedersen O. Gut microbiota in patients with type 2 diabetes mellitus. European Journal of Endocrinology 2015; 172, R167-R177.

8. Frazier TH, DiBaise JK and McClain CJ. Gut microbiota, intestinal permeability, obesity-induced inflammation and liver injury. JPEN J Parenter Enteral Nutr. 2011;35 5 Suppl:14S---20S.

9. Reigstad CS , Kashyap PC. Beyond phylotyping: understanding the impact of gut microbiota on host biology. Neurogastroenterol Motil 2013; 25: 358-372.

10. Tilg H , Kaser A. Gut microbiome, obesity, and metabolic dysfunction. Journal of Clinical Investigation 2011;121: 2126-2132.

11. Tremaroli V, Bäckhed F. Functional interactions between the gut microbiota and host metabolism. Nature 2012;489 : 242-249.

12. Cani PD, Delzenne NM. The Role of the Gut Microbiota in Energy Metabolism and Metabolic Disease. Current Pharmaceutical Design 2009; 15, 1546-1558.

13. Stappenbeck TS, Hooper LV, Gordon JI. Developmental regulation of intestinal angiogenesis by indigenous microbes via Paneth cells. Proc Natl acad Sci USA 2002; 99:15451-15455. 
14. Ba“ckhed F, Ding H, Wang T, Hooper LV, Koh GY, Nagy A, et al. The gut microbiota as an environmental factor that regulates fat storage. Proc Natl Acad Sci U S A 2004;101: 1571815723.

15. Denechaud PD, Dentin R, Girard J , Postic C. Role of ChREBP in hepatic steatosis and insulin resistance. FEBS Lett 2008; 582: 68- 73.

16. Serino M, Fernandez-Real JM, García-Fuentes E, Queipo-Ortuño M, Moreno-Navarrete JM, Sánchez A, et al. The gut microbiota profile is associated with insulin action in humans. Acta Diabetol 2012; DOI:10.1007/s00592-012-0410-5.

17. Bäckhed F, Manchester JK, Semenkovich CF, Gordon JI. Mechanisms underlying the resistance to diet-induced obesity in germ-free mice. Proc Natl Acad Sci USA 2007; 104:979-84.

18. Samuel B S, Shaito A, Motoike T, Rey FE, Backhed F, Manchester JK, et al. Effects of the gut microbiota on host adiposity are modulated by the short-chain fatty-acid binding $\mathrm{G}$ proteincoupled receptor, Gpr41. Proc. Natl Acad. Sci. USA 2008; . 105, 16767-16772.

19. Lee YH, Giraud J, Davis RJ, White MF. c-jun $N$ terminal kinase (JNK) mediates feedback inhibition of the insulin signaling cascade. $J$. Biol. Chem. 2003; 278, 2896-2902.

20. Bjursell M, Admyre T, Göransson M, Marley AE, Smith DM, Oscarsson J, et al. Improved glucose control and reduced body fat mass in free fatty acid receptor 2 (Ffar2) deficient mice fed a high fat diet. Am. J. Physiol. Endocrinol. Metab 2011; 300, E211-E220.

21. Geurts L, Lazarevic V, Derrien M, Everard A, Van Roye M, Knauf C, et al.. Altered gut microbiota and endocannabinoid system tone in obese and diabetic leptin-resistant mice: impact on apelin regulation in adipose tissue. Frontiers in Microbiology 2011; 2: 149.

22. Feuerer M, Herrero L, Cipolletta D, Naaz A, Wong J, Nayer A, et al. Lean, but not obese, fat is enriched for a unique population of regulatory $\mathrm{T}$ cells that affect metabolic parameters. Nat Med 2009; 15: 930-939.

23. Erridge C, Attina T, Spickett CM, Webb DJ. A high-fat meal induces low-grade endotoxemia: evidence of a novel mechanism of postprandial inflammation. Am J Clin Nutr. 2007 Nov; 86(5): 1286-92.

24. Ghoshal S, Witta J, Zhong J, de Villiers W, Eckhardt E. Chylomicrons promote intestinal absorption of lipopolysaccharides. J. Lipid Res. 2009; 50, 90-97.

25. Brun P, Castagliuolo I, di Leo V, Buda A, Pinzani $\mathrm{M}$, Palù $\mathrm{G}$, et al. Increased intestinal permeability in obese mice: New evidence in the pathogenesis of nonalcoholic steatohepatitis. Am. J. Physiol. Gastrointest. Liver Physiol. 2007; 292, G518G525.
26. Silvestri C and Di Marzo V. The endocannabinoid system in energy homeostasis and the etiopathology of metabolic disorders. Cell Metab 2013; 17: 475-490.

27. Côté M, Matias I, Lemieux I, Petrosino S, Alméras N, Després JP, et al. Circulating endocannabinoid levels, abdominal adiposity and related cardiometabolic risk factors in obese men. Int J Obes 2007; 31: 692-699.

28. de La Serre CB, Ellis CL, Lee J, Hartman A L, Rutledge J C , Raybould H. E. Propensity to high-fat diet induced obesity in rats is associated with changes in the gut microbiota and gut inflammation. Am J Physiol 2010; 299: G440-448.

29. Everard A, Belzer C, Geurts L, Ouwerkerk JP, Druart C, Bindels LB, et al. Cross-talk between Akkermansia muciniphila and intestinal epithelium controls diet-induced obesity. Proc Natl Acad Sci USA 2013; 110: 9066-9071.

30. Festi D, Schiumerini R, Eusebi LH, Marasco G, Taddia M , Colecchia A. Gut microbiota and metabolic syndrome. World J Gastroenterol 2014; 20(43): 16079-16094.

31. Cani PD, Amar J, Iglesias MA, Poggi M, Knauf C, Bastelica D, et al. Metabolic endotoxemia initiates obesity and insulin resistance. Diabetes 2007; 56(7):1761-1772.

32. Akira S, Uematsu S, Takeuchi O. Pathogen recognition and innate immunity. Cell 2006, 124, 783-801.

33. Tsukumo DM, Carvalho-Filho MA, Carvalheira JB, Prada PO, Hirabara SM, Schenka AA, et al. Loss-of-function mutation in Toll-like receptor 4 prevents diet-induced obesity and insulin resistance. Diabetes 2007; 56, 1986-1998.

34. Carvalho-Filho MA, Ueno M, Hirabara SM, Seabra $\mathrm{AB}$, Carvalheira JB, de Oliveira $\mathrm{MG}$, et al. $S$ nitrosation of the insulin receptor, insulin receptor substrate 1, and protein kinase B/Akt: A novel mechanism of insulin resistance. Diabetes 2005; 54, 959-967.

35. Caricilli AM, Nascimento PH, Pauli JR, Tsukumo DM, Velloso LA, Carvalheira JB, et al. Inhibition of Toll-like receptor 2 expression improves insulin sensitivity and signaling in muscle and white adipose tissue of mice fed a high-fat diet. $J$. Endocrinol. 2008; 199, 399-406.

36. Rabot S, Membrez M, Bruneau A, Gérard P, Harach T, Moser M, et al. Germ-free C57BL/6J mice are resistant to high-fat-diet-induced insulin resistance and have altered cholesterol metabolism. FASEB J. 2010; 24, 4948-4959.

37. Schertzer JD and Klip A. Give a NOD to insulin resistance. Am. J. Physiol. Endocrinol. Metab. 2011; 301, E585-E586.

38. Zhao L, Hu P, Zhou Y, Purohit J and Hwang D. NOD1 activation induces proinflammatory gene expression and insulin resistance in 3T3-L1 adipocytes. Am. J. Physiol. Endocrinol. Metab. 2011; 301, E587-E598. 
39. Puddu A, Sanguineti R, Montecucco F and Viviani GL. Evidence for the Gut Microbiota Short-Chain Fatty Acids as Key Pathophysiological Molecules Improving Diabetes. Hindawi Publishing Corporation. Mediators of Inflammation 2014; Article ID 162021, 9.

40. Lau E, Carvalho D, Pina-Vaz C, Barbosa JA, Freitas P. Beyond gut microbiota: understanding obesity and type 2 diabetes. Hormones 2015; 14(3):358-369.

41. Koeth RA, Levison BS, Culley MK, Buffa JA, Wang Z, Gregory JC, et al. Gamma-butyrobetaine is a proatherogenic intermediate in gut microbial metabolism of L-carnitine to TMAO. Cell Metab. 2014;20:799-812.

42. Queipo-Ortuño MI, Seoane LM, Murri M, Pardo M, Gomez-Zumaquero JM, Cardona F, et al. Gut microbiota composition in male rat models under different nutritional status and physical activity and its association with serum leptin and ghrelin levels. PLoS One. 2013, 28;8(5):e65465.

43. Kang SS, Jeraldo PR, Kurti A, Miller ME, Cook $\mathrm{MD}$, Whitlock $\mathrm{K}$, et al. Diet and exercise orthogonally alter the gut microbiome and reveal independent associations with anxiety and cognition. Mol Neurodegener. 2014;9:36.

44. Carvalho BM, Guadagnini D, Tsukumo DM, Schenka AA, Latuf-Filho P, Vassallo J, et al. Modulation of gut microbiota by antibiotics improves insulin signalling in high-fat fed mice. Diabetologia. 2012;55:2823-34.

45. Trasande L, Blustein J, Liu M, Corwin E, Cox LM , Blaser MJ. Infant antibiotic exposures and early-life body mass. Int J Obes (Lond). 2013; 37:16-23.

46. Mueller NT, Whyatt R, Hoepner L, Oberfield $\mathrm{S}$, Dominguez-Bello MG, Widen EM, et al. Prenatal exposure to antibiotics, cesarean section, and risk of childhood obesity. Int J Obes (Lond). 2014.

47. Vrieze A, Out C, Fuentes S, Jonker L, Reuling I, Kootte RS, et al. Impact of oral vancomycin on gut microbiota, bile acid metabolism, and insulin sensitivity. Journal of Hepatology 2014; 60 824831.

48. Jernberg C, Lofmark S, Edlund C , Jansson JK. Long-term ecological impacts of antibiotic administration on the human intestinal microbiota. ISME J. 2007;1:56-66.

49. Han JL, Lin HL. Intestinal microbiota and type 2 diabetes: from mechanism insights to therapeutic perspective. World J Gastroenterol 2014; 20(47): 17737-17745.

50. Liou AP, Paziuk M, Luevano JM, Machineni S, Turnbaugh PJ and Kaplan LM. Conserved shifts in the gut microbiota due to gastric bypass reduce host weight and adiposity. Sci Transl Med. 2013; 5:178ra141.
51. Li JV, Ashrafian H, Bueter M, Kinross J, Sands $\mathrm{C}$, le Roux CW, et al. Metabolic surgery profoundly influences gut microbial-host metabolic crosstalk. Gut 2011; 60: 1214-1223.

52. Hill C, Guarner F, Reid G, Gibson GR, Merenstein DJ, Pot B, et al. Expert consensus document: The International Scientific Association for Probiotics and Prebiotics consensus statement on the scope and appropriate use of the term probiotic. Nat Rev Gastroenterol Hepatol 2014; 11: 506_14.

53. Thygesen JB, Glerup H and Tarp B. Saccharomyces boular diifungemia caused by treatment with a probioticum. BMJ Case Rep 2012; doi:10.1136/bcr.06. 2011.4412.

54. Ejtahed HS, Mohtadi-Nia J, Homayouni-Rad A, Niafar M, Asghari-Jafarabadi M and Mofid V. Probiotic yogurt improves antioxidant status in type 2 diabetic patients, Nutrition 2012;28 : 539543.

55. Roberfroid M, Gibson GR, Hoyles L, McCartney AL, Rastall R, Rowland I, et al. Prebiotic effects: metabolic and health benefits. British Journal of Nutrition 2010;104: S1-S63.

56. Roberfroid M. Prebiotics: the concept revisited. J Nutr 2007; 137: 830S_7S.

57. Parnell JA, Reimer RA. Prebiotic fibres dosedependently increase satiety hormones and alter Bacteroidetes and Firmicutes in lean and obese JCR: LA-cp rats. Br J Nutr 2012; 107: 601-613.

58. Neyrinck AM, Possemiers S, Druart C, Van de Wiele T, De Backer F, Cani PD, et al. Prebiotic effects of wheat arabinoxylan related to the increase in bifidobacteria, Roseburia and Bacteroides/ Prevotella in diet-induced obese mice. PLoS One 2011; 6: e20944.

59. Macfarlane G, Steed H, Macfarlane S. Bacterial metabolism and health-related effects of galactooligosaccharides and other prebiotics. J Appl Microbiol 2008; 104: 305_44.

60. Guarner F. Studies with inulin-type fructans on intestinal infections, permeability, and inflammation. J Nutr 2007; 137:2568S-2571S.

61. Cani PD, Neyrinck AM, Fava F, Knauf C, Burcelin RG, Tuohy KM, et al. Selective increases of bifidobacteria in gut microflora improve high-fatdiet-induced diabetes in mice through a mechanism associated with endotoxaemia. Diabetologia 2007; 50: 2374-2383.

62. Vrieze A, Van Nood E, Holleman F, Salojärvi J, Kootte RS, Bartelsman JF, et al. Transfer of intestinal microbiota from lean donors increases insulin sensitivity in individuals with metabolic syndrome. Gastroenterology 2012; 143(4):913. 
Peer reviewer: Rashed Hasan, Professor of Tropical Medicine Hepatogastroenterology, Faculty of Medicine, Zagazig University, Egypt.
Editor: Tarik Zaher, Professor of Tropical Medicine and Hepatogastroenterology,Faculty of Medicine, Zagazig University, Egypt. 